\title{
EFECTO DE TRES TENSIONES DE HUMEDAD DEL SUELO SOBRE LA GERMINACIÓN DE SEMILLAS DE DIFERENTES TAMAÑOS EN MAÍZ (Zea mays L.)
}

\section{EFFECT OF THREE MATRIC SOIL POTENTIALS ON SEED GERMINATION OF DIFFERENT SIZES OF TWO CORN (Zea mays L.) CULTIVARS}

José A. Laynez-Garsaball1', Jesús Rafael Méndez Natera y Juliana Mayz-Figueroa

Recibido para evaluación: Agosto 15 de 2007 - Aceptado para publicación: Enero 20 de 2008

\section{RESUMEN}

Se estudio el efecto de tres frecuencias de riego que produjeron tres niveles de tensión de humedad en el suelo sobre la germinación de semillas de maíz. Se estimó la curva de retención de humedad del suelo y mediante análisis de regresión, las ecuaciones de ajuste de las rectas y el rango de fluctuación en la tensión del agua del suelo dentro de cada intervalo de riego para cada frecuencia establecida. La siembra se efectuó en bandejas de aluminio, $10 \mathrm{~kg}$ suelo bandeja ${ }^{-1}$ y 50 semillas. El diseño estadístico fue parcelas divididas con cuatro repeticiones, parcela principal: tensiones de humedad del suelo $(0,-0,6$ y - 1,2 MPa), subparcelas: dos cultivares de maíz (Himeca 95 y Pioneer 3031), y sub-subparcelas: tres tamaños de semillas (TS) (<0.32 g; 0.32, 0.36 g, y > 0.36 g). Se evaluó: porcentaje de germinación (\%), índice de velocidad de germinación (IVG) y número medio de días a germinación (NMDG). Fue posible discriminar entre cultivares para esta condición de estrés en el proceso de germinación con una tensión de la humedad del agua en el suelo de -1,2 MPa (Pioneer 3031 superior a Himeca 95). El tamaño de la semilla no influenció el proceso de germinación.

Palabras clave: Sequía en suelo, potencial osmótico, frecuencia de riego

\begin{abstract}
Seed germination was studied from three seed sizes of two corn cultivars under three irrigation intervals which produced three matric potentials. Soil moisture retention curves were determined and the fit equations of lines were estimated by regression analysis in order to calculate the fluctuation range of

${ }^{1}$ Universidad de Oriente. Escuela de Ingeniería Agronómica. Núcleo Monagas. Avenida Universidad. Campus Los Guaritos. Maturín, 6201. Monagas, Venezuela. Email: jalaynezg@yahoo.es

${ }^{2}$ Universidad de Oriente. Postgrado en Agricultura Tropical. Núcleo Monagas, Campus Juanico. Maturín, 6201.
\end{abstract}


soil water pressure in each irrigation interval for each frequency established. Sowing was carried out in aluminum trays with $10 \mathrm{~kg}$ of soil tray ${ }^{-1}$ and 50 seeds. A split-split-plot design was used with four replications, main plots were the osmotic potentials $(0.0,-0.6$ and $-1.2 \mathrm{MPa})$, sub-plots were the corn cultivars (Himeca 95 and Pioneer 3031) and sub-sub-plots were seed sizes (SS) (<0.32 g; 0.32, $0.36 \mathrm{~g} \mathrm{y}>0.36 \mathrm{~g}$ ). The following evaluations were recorded: germination percentage, germination speed index and days to total germination. It was possible to discriminate for drought tolerance in germination stage with osmotic potentials of - 1.2 MPa (cultivar Pioneer 3031 was more tolerant than Himeca 95). Seed size did not affect the germination process.

Key words: Soil drought, osmotic potential, irrigation frequency.

\section{INTRODUCCIÓN}

El estrés hídrico es el más importante que pueden sufrir las plantas, y especialmente, los cultivos en cuanto a la cantidad de materia vegetal afectada. Las pérdidas agrícolas ocasionadas por el estrés hídrico son superiores al conjunto de las pérdidas producidas por los restantes estreses (AzconBieto y Talon, 1993). La sequía, a nivel de los trópicos, afecta la producción agrícola en casi un $60 \%$ de las tierras. En el cultivo del maíz, las sequías reducen los rendimientos en cerca de $15 \%$ anualmente en las tierras bajas tropicales y subtropicales, Ilegando a causar pérdidas estimadas en 16 millones ton de grano (Edmeades et al., 1992). La sequía en la germinación limita la población de plantas y en floración (espigamiento) es más perjudicial para el rendimiento de granos.

Uno de los principales problemas que enfrenta la agricultura de secano es la sequía, la cual algunas veces restringe la emergencia y el desarrollo inicial de las plántulas. El estrés hídrico puede resultar en el retraso y la reducción de la germinación de las semillas o puede restringir la germinación completamente (Turk et al., 2004). Las pruebas de calidad de semillas se dirigen a tratar de predecir la vida de almacenamiento de un lote de semillas o su calidad después de un período especificado, la emergencia en campo después de la siembra, y el subsiguiente vigor de las plántulas y el rendimiento final del cultivo (Ellis y Roberts, 1980). La técnica mayormente usada es la del envejecimiento acelerado; sin embargo, es cuestionada por la falta de estandarización en diferencias en humedad de la semilla, temperatura y duración del tratamiento (Musgrove et al., 1980). Pequeñas diferencias en el tratamiento de envejecimiento usado dan lugar a variaciones en el vigor. Aparte de mencionar, que la técnica de envejecimiento acelerado no permite estudiar el efecto del estrés por sequía. Otra técnica para la identificación de semillas de calidad en diferentes lotes almacenados, y que al mismo tiempo permite estudiar el efecto del estrés hídrico, de una manera sencilla, no costosa y sin requerir de equipos especializados, consiste en la siembra de semillas de distintos lotes almacenados o cultivares, en condiciones de umbráculo, usando como sustrato suelo sometido a diversas frecuencias de riego.

El tamaño de la semilla induce variaciones en la germinación y en el crecimiento de las plántulas dentro de un mismo genotipo, atribuidas a la influencia del tamaño de la semilla en la tolerancia a estas condiciones, como lo demuestra la investigación sobre el efecto del estrés por sequía en genotipos de maíz de Muchena y Grogan (1977). Esto sugiere la posible selección de un determinado tamaño de semilla de un cultivar con objeto de brindar mayor tolerancia al estrés bien sea 
durante la germinación, o bien, durante los primeros días de crecimiento de las plántulas. Gazanchian et al. (2006) evaluó el uso de cuatro niveles de agua en el suelo sobre la emergencia de plántulas de 36 poblaciones de gramíneas nativas de temporada fría de 15 especies de siete géneros (Elymus, Agropyron, Secale, Hordeum, Bromus, Festuca y Dactylis) colectadas en diferentes localidades de Irán. Estos autores en un experimento de invernadero, inmediatamente después de la siembra, añadieron agua al suelo seco para alcanzar la capacidad de campo y 25, 50 y $75 \%$ de la misma, los cuales dieron aproximadamente los potenciales mátricos del suelo de -0.03; -0.2; - 0.6 y - $15 \mathrm{MPa}$ (cercano al punto de marchitez permanente), respectivamente. Los autores concluyeron que el método de evaluación usado pudiera efectivamente detectar variaciones en la emergencia de plántulas de gramíneas perennes de época fría causadas por una baja humedad del suelo. Las gramíneas exhibieron una alta diversidad genética para la emergencia, la tasa de emergencia y el crecimiento de raíces y vástagos bajo condiciones de baja humedad del suelo y que considerable variación en la respuesta a esta baja humedad del suelo ocurrió entre y dentro las especies y poblaciones colectadas. En otro experimento, Cornaglia et al. (2005) utilizaron solamente un lote de semillas de Dallisgrass (Paspalum dilatatum Poir.), comercialmente producido en Australia, para estudiar el efecto de regímenes de agua que simularon el amplio rango de condiciones de humedad del suelo que pueden ocurrir durante la estación en los periodos de emergencia. Para ello aplicaron cuatro tratamientos: capacidad de campo continua y tres frecuencias de riego, cada 2, 4 y 7 días, y para todos los tratamientos, la subirrigación fue provista durante unos pocos minutos hasta que la superficie del suelo pareció húmeda. Los autores concluyeron que el patrón de la disponibilidad de agua en el suelo reduce el marco temporal bajo el cual el establecimiento exitoso de esta especie pudiera ser posible, excepto en hábitats muy húmedos, lo que indica la eficiencia del método utilizado por ellos. Turk et al. (2004) encontraron que una disminución del potencial osmótico de 0 a -0.33 MPa condujo a una ligera reducción significativa en la germinación en todos los cultivares de lenteja (Lenus culinaris Medic) excepto en FLIP 8616, un cultivar tolerante a la sequía. La reducción en el porcentaje de germinación puede ser atribuida a una menor difusión del agua en las cubiertas de las semillas a un mayor estrés hídrico. Este experimento fue planificado para evaluar el efecto de tres frecuencias de riego que produjeron tres niveles de tensión de humedad en el suelo sobre la germinación de plántulas a partir de tres tamaños de semilla de dos cultivares de maíz, en condiciones de umbráculo.

\section{MATERIALES Y MÉTODOS}

\section{Ubicación}

La presente investigación se llevo a cabo en el umbráculo del Postgrado en Agricultura Tropical de la Universidad de Oriente, Estado, Monagas, Venezuela.

\section{Variables medidas}

Las respuestas de los cultivares de maíz a los distintos tratamientos fueron evaluadas a través de los siguientes parámetros:

- Porcentaje de germinación (\%): a los 4, 8, 12 y 16 días después de la siembra (dds).

- Índice de velocidad de germinación (IVG): estimado de acuerdo con la metodología de Khan y Ungar, (1984) a través de la fórmula: IVG $=\left(N_{1} \times 4+N_{2} \times 4+\ldots+N_{n} \times\right) /$ $\mathrm{T}_{\mathrm{n}}$ )

- Número medio de días a germinación (NMDG): determinado con base en la 
EFECTO DE TRES TENSIONES DE HUMEDAD DEL SUELO SOBRE LA GERMINACIÓN DE SEMILLAS. .

metodología de Hartmann et al. (1993) por la fórmula:

$\mathrm{NMDG}=\left(\mathrm{N}_{1} \times \mathrm{T}_{1}+\mathrm{N}_{2} \times \mathrm{T}_{2}+\ldots+\mathrm{N}_{\mathrm{n}} \times\right.$ $\left.\left.\mathrm{T}_{\mathrm{n}}\right) / \mathrm{n}\right)$

$\mathrm{N}=$ número de semillas germinadas dentro de los intervalos de tiempo consecutivos.

$\mathrm{T}=$ tiempo transcurrido entre el inicio de la prueba y el fin del intervalo.

$\mathrm{n}=$ número de semillas germinadas .

\section{Procedencia del suelo}

Se usó suelo no esterilizado (previamente secado al aire y cernido a través de un tamiz de malla de $3 \mathrm{~mm}$ ), obtenido de una franja entre los 5 y $20 \mathrm{~cm}$ de profundidad proveniente de las parcelas experimentales del Campus Los Guaritos de la Universidad de Oriente, Maturín, Estado Monagas, Venezuela. Las características físicas y químicas del suelo se determinaron a través del análisis respectivo en el Laboratorio de Servicios Agroambientales (LABSEA) de la Universidad de Oriente. El suelo usado presentó textura franco arenosa, un contenido medio de $\mathrm{P}\left(15,9 \mathrm{mg} \mathrm{kg}^{-1}\right)$, alto de calcio $\left(1,01 \mathrm{cmol} \mathrm{kg}^{-1}\right)$ y bajo de magnesio

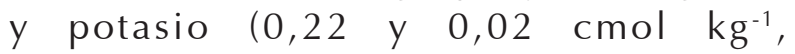
respectivamente), bajo en materia orgánica $(0,86 \%)$ y $\mathrm{pH}$ ácido $(5,0)$.

\section{Determinación del rango de fluctuación en la tensión del agua en el suelo}

Al suelo se le realizó una estimación de la curva de retención de humedad empleando la metodología de las ollas de presión ó método de Richard (1967) a $0.033 ; 0.1 ; 0.3 ; 1.0$ y 1.5 MPa. Con los valores obtenidos se calcularon mediante análisis de regresión las ecuaciones de ajuste de las rectas. Se estimó el rango de fluctuación en la tensión del agua del suelo dentro de cada intervalo de riego para cada frecuencia establecida. Para ello, se determinó diariamente el contenido de humedad de cada unidad experimental, a la misma hora, por diferencia en peso entre el valor correspondiente a capacidad de campo y el valor obtenido al momento de evaluar. Se estableció la equivalencia del contenido de humedad del suelo en términos de tensión del agua en el suelo mediante la ecuación que mejor ajustó de acuerdo con la curva de retención de humedad previamente determinada. Una vez determinada la tensión del agua en el suelo para cada unidad experimental se efectuó la aplicación del riego a capacidad de campo a aquellas unidades que así correspondía conforme a las frecuencias de riego establecidas.

\section{Cultivares y cribaje de la semilla}

Se utilizó semilla certificada de los cultivares comerciales de maíz: Himeca 95 y Pioneer 3031, con un contenido de humedad promedio de $12 \%$, tratadas con Vitavax 200 $\mathrm{F}^{\circledR}$ (carboxin 17\% + thiram 17\%), para prevenir el crecimiento de hongos durante la germinación. Dos kilogramos de semillas de cada cultivar fueron sometidos a un proceso de selección por el que las semillas se separaron con base al peso individual, en tres intervalos de peso, en lotes de 300 semillas: < $0.32 \mathrm{~g}, 0.32-0.36 \mathrm{~g}$ y $>036 \mathrm{~g}$.

\section{Siembra y diseño experimental}

Se emplearon bandejas de aluminio $(41 \mathrm{~cm}$ largo, $26.5 \mathrm{~cm}$ ancho y $10 \mathrm{~cm}$ alto), desinfectadas con hipoclorito de sodio (5.25\% de cloro activo) y posteriormente lavadas con abundante agua para eliminar el exceso de desinfectante. Cada bandeja tuvo $10 \mathrm{~kg}$ de suelo y 50 semillas arregladas en lotes de 25 de acuerdo al tratamiento correspondiente (repetición, sustrato, cultivar, tamaño de la semilla), distribuidas en cinco hileras de cinco semillas cada una (distancia entre plantas 3.7 $\mathrm{cm}$ y entre semillas $4 \mathrm{~cm}$ ) y a $3 \mathrm{~cm}$ de profundidad. Se efectuaron riegos diarios de $250 \mathrm{ml}$ agua por bandeja.

Se empleó el diseño estadístico de parcelas 
divididas con cuatro repeticiones, donde la parcela principal la conformaron las tensiones de humedad del suelo (capacidad de campo (Testigo), -0,6 y -1,2 MPa), ocasionadas por riego cada 24, 48 y 72 horas, respectivamente. Las subparcelas, Ios dos cultivares de maíz (Himeca 95 y Pioneer 3031), y las sub-subparcelas, los tres tamaños de las semillas $(<0.32 \mathrm{~g} ; 0.32-0,36 \mathrm{~g}$ y $>$ $036 \mathrm{~g})$.

Los resultados de los parámetros evaluados fueron analizados mediante el análisis de varianza convencional. Los datos del porcentaje de germinación fueron transformados mediante la fórmula $\sqrt{(X+3 / 8) /(n+3 / 4)}$ para el porcentaje a los 4 dds y $\sqrt{X}$ para los porcentajes de germinación a los 8, 12 y 16 dds. Las diferencias entre las medias se establecieron a través de la Prueba de Rangos Múltiples de Duncan al 5\% de probabilidad.

\section{RESULTADOS Y DISCUSIÓN}

\section{Porcentaje de germinación}

Los análisis de varianza para el porcentaje de germinación a los 4, y 8, 12 y 16 dds $(\operatorname{Pr}<0.05)$ únicamente señalan efecto significativo para los efectos simples potencial osmótico y cultivar de maíz, y para la interacción potencial osmótico x cultivar de maíz a los 4 dds y para la interacción potencial osmótico $x$ cultivar de maíz x tamaño de la semilla a los 8,12 y 16 dds. Al analizar la interacción potencial osmótico x cultivar a los 4 dds (Tabla 1), la menor germinación ocurrió en ambos cultivares en el potencial osmótico de -1.2 $\mathrm{MPa}$, y la mayor en los potenciales del testigo y -0,6 MPa, estos últimos estadísticamente similares. Al analizar entre cultivares a un mismo potencial osmótico, Pioneer 3031 presentó mayor germinación que Himeca 95 únicamente en el menor potencial osmótico (-1.2 MPa). Al estudiar la doble interacción potencial osmótico x cultivar x tamaño de la semilla a los 8 dds (Tabla 2), la cual es exactamente igual para aquella a los 12 y 16 dds, se observa que la germinación fue menor únicamente en el potencial osmótico testigo con el cultivar Himeca 95, en el menor tamaño de semilla con respecto al cultivar Pioneer 3031, el resto de los tratamientos fueron similares entre sí. Al comparar los potenciales osmóticos, se encontró que la menor germinación ocurrió en el potencial del testigo en comparación con los potenciales de -0.6 y -1.2 MPa para el cultivar Himeca 95 y el menor tamaño de la semilla, el resto de los tratamientos fueron similares entre si. En relación al tamaño de la semilla, las grandes y medianas, tuvieron una mayor germinación que las de menor tamaño en el cultivar Himeca 95 y el potencial osmótico testigo, el resto de los tratamientos entre los tamaños de semillas fueron similares entre sí.

Tabla 1. Promedios para el porcentaje de germinación a los cuatro días después de la siembra de tres tamaños de semilla de dos cultivares de maíz (Zea mays L.), bajo tres tensiones de humedad del suelo.

\begin{tabular}{ccc}
\hline $\begin{array}{c}\text { Potenciales osmóticos } \\
\text { (MPa) }\end{array}$ & \multicolumn{3}{c}{ Cultivares } \\
\hline Testigo & Himeca $\mathbf{9 5}$ & Pioneer $\mathbf{3 0 3 1}$ \\
-0.6 & $97.63 \mathrm{Aa}^{*}$ & $99.32 \mathrm{Aa}$ \\
-1.2 & $96.97 \mathrm{Aa}$ & $99.32 \mathrm{Aa}$ \\
\hline
\end{tabular}

*Prueba de Rangos Múltiples de Duncan (Pr<0.05), letras mayúsculas para las comparaciones verticales, letras minúsculas para las comparaciones horizontales y letras diferentes indican promedios estadísticamente diferentes. 
Tabla 2. Promedios para el porcentaje de germinación a los ocho días después de la siembra de tres tamaños de semilla de dos cultivares de maíz (Zea mays L.), bajo tres tensiones de humedad del suelo.

\begin{tabular}{cccrr}
\hline \multirow{2}{*}{ Genotipos } & $\begin{array}{c}\text { Potenciales } \\
\text { osmóticos (MPa) }\end{array}$ & $\mathbf{< 0 . 3 2 5}$ & $\mathbf{0 . 3 2} \mathbf{- 0 . 3 6}$ & \multicolumn{1}{c}{ Tamaños de las semillas (g) } \\
\hline \multirow{3}{*}{ Himeca 95 } & Testigo & $95.981 \mathrm{BbY}^{*}$ & $100.000 \mathrm{AaX}$ & $100.000 \mathrm{AaX}$ \\
& -0.6 & $100.000 \mathrm{AaX}$ & $100.000 \mathrm{AaX}$ & $99.980 \mathrm{AaX}$ \\
& -1.2 & $99.980 \mathrm{ABaX}$ & $97.911 \mathrm{AaX}$ & $97.990 \mathrm{AaX}$ \\
Pioneer 3031 & Testigo & $100.000 \mathrm{AaX}$ & $97.990 \mathrm{AaX}$ & $100.000 \mathrm{AaX}$ \\
& -0.6 & $100.000 \mathrm{AaX}$ & $99.980 \mathrm{AaX}$ & $100.000 \mathrm{AaX}$ \\
& -1.2 & $97.990 \mathrm{AaX}$ & $100.000 \mathrm{AaX}$ & $99.980 \mathrm{AaX}$ \\
\hline
\end{tabular}

*Prueba de Rangos Múltiples de Duncan $(P r<0,05)$, letras mayúsculas (A, B y C) para comparaciones entre potenciales osmóticos a un mismo nivel de tamaño de semilla y cultivar, letras minúsculas para la comparación de los diferentes tamaños de semilla a un mismo potencial osmótico y cultivar, letras mayúsculas (X y Y) para comparaciones entre cultivares a un mismo nivel de potencial osmótico y tamaño de semilla.

\section{Índice de velocidad de germinación (IVG)}

El análisis de varianza para el índice de velocidad la germinación basado en los porcentajes de germinación a los 4, 8, 12 y 16 dds $(P r<0.05)$, solo encontró diferencias significativas $(\operatorname{Pr}<0.05)$ para los efectos simples potencial osmótico y cultivar de maíz, y para la interacción potencial osmótico $x$ cultivar de maíz. Al analizar la interacción potencial osmótico x cultivar (Tabla 3) el IVG fue menor en ambos cultivares en el potencial osmótico menor (-1.2 MPa), siendo el testigo y el potencial osmótico de -0.6 MPa estadísticamente iguales. Al comparar entre cultivares para cada nivel de potencial osmótico el menor IVG fue para Pioneer 3031 en el menor potencial osmótico, en los otros potenciales no existieron diferencias estadísticas entre cultivares.

\section{Número medio de días a germinación (NMDG)}

El análisis de varianza para el número medio de días a germinación únicamente reportó significación estadística para los factores simples potencial osmótico y cultivar de maíz $(\operatorname{Pr}<0.05)$. En la prueba de separación de medias para el factor potencial osmótico (Tabla 4), las semillas tardaron menor tiempo en germinar en el testigo, seguidas por aquellas sometidas al potencial osmótico de -0.6 Mpa, las cuales a su vez germinaron más

Tabla 3. Promedios para el índice de velocidad de germinación después de la siembra de tres tamaños de semilla de dos cultivares de maíz (Zea mays L.), bajo tres tensiones de humedad del suelo.

\begin{tabular}{|c|c|c|}
\hline \multirow{2}{*}{$\begin{array}{c}\text { Potenciales osmóticos } \\
\text { (MPa) }\end{array}$} & \multicolumn{2}{|c|}{ Cultivares } \\
\hline & Himeca 95 & Pioneer 3031 \\
\hline Testigo & 24.592 Аа* $^{*}$ & $24.833 \mathrm{Aa}$ \\
\hline-0.6 & 24.583 Аа & $24.875 \mathrm{Aа}$ \\
\hline-1.2 & $15.667 \mathrm{Bb}$ & $19.333 \mathrm{Ba}$ \\
\hline
\end{tabular}

* Prueba de Rangos Múltiples de Duncan $(P r<0.05)$, letras mayúsculas para las comparaciones verticales, letras minúsculas para las comparaciones horizontales, letras diferentes indican promedios estadísticamente diferentes. 
TEMAS AGRARIOS - Vol. 13:(1) Enero - Junio 2008 (14 - 24)

Tabla 4. Promedios para los números medios de días a germinación después de la siembra de tres tamaños de semilla de dos cultivares de maíz (Zea mays L.), bajo tres tensiones de humedad del suelo.

\begin{tabular}{|c|c|}
\hline Potenciales Osmóticos (MPa) & Número medio de días a germinación \\
\hline Testigo & $3.212 \mathrm{~A}^{*}$ \\
\hline-0.6 & $3.432 \mathrm{~B}$ \\
\hline-1.2 & $4.589 \mathrm{C}$ \\
\hline
\end{tabular}

*Prueba de Rangos Múltiples de Duncan $(\operatorname{Pr}<0.05)$, letras diferentes indican promedios estadísticamente diferentes.

rápidamente que aquellas a -1.2 Mpa. En la prueba de medias para el factor cultivar, el menor NMDG correspondió a Pioneer 3031 (3.57 días) con respecto a Himeca 95 (3.92 días). En este ensayo en el que se utilizó suelo con diferentes tensiones del agua logradas con distintas frecuencias de riego, aun cuando se presentó una doble interacción potencial $x$ cultivar x tamaño en la germinación a los 8 dds, la misma no tiene importancia desde el punto de vista de su posible utilidad para la selección de cultivares tolerantes a la sequía dado que indicó que la germinación fue menor en el potencial testigo que en el resto de los tratamientos solo para el cultivar Pioneer 3031, en el menor tamaño de semilla. Sin embargo, existieron interacciones potencial $x$ cultivar en el porcentaje de germinación a los 4 dds y en el IVG, señalando la posibilidad de discriminar entre cultivares para tolerancia a sequía si se trabaja con potenciales de agua en el suelo iguales a -1.2 $\mathrm{MPa}$, y probablemente por encima de este valor. Ávila et al. (2007) encontraron que la prueba de la longitud de las plántulas bajo estrés hídrico de -0,9 Mpa fue la mejor para evaluar el potencial fisiológico de las semillas de maíz.

La utilización de la metodología para generar los diferentes potenciales osmóticos en este ensayo fue efectiva a la hora de discriminar entre los dos genotipos para su tolerancia a la sequía. Resultados similares fueron reportados por Gazanchian et al. (2006) en un ensayo con 36 poblaciones de gramíneas de 15 especies de siete géneros (Elymus, Agropyron, Secale, Hordeum, Bromus, Festuca y Dactylis) en Irán trabajando con potenciales mátricos del suelo de $-0.03,-0.2 ;-0.6$ y - $1.5 \mathrm{MPa}$, y concluyeron que el método de evaluación usado pudiera efectivamente detectar variaciones en la emergencia de plántulas de gramíneas perennes de época fría causadas por una baja humedad del suelo. En otro experimento, Cornaglia et al. (2005) utilizaron un lote de semillas de Dallisgrass (Paspalum dilatatum Poir.) en Australia para estudiar el efecto de regímenes de agua, para ello aplicaron cuatro tratamientos: capacidad de campo continua y tres frecuencias de riego, cada 2, 4 y 7 días, y concluyeron que el patrón de la disponibilidad de agua en el suelo reduce el marco temporal bajo el cual el establecimiento exitoso de esta especie pudiera ser posible, excepto en habitáts muy húmedos, lo que indica la eficiencia del método utilizado por ellos.

En el ensayo se observó, en general, una disminución en la germinación por efecto del incremento del estrés hídrico al inicio de la germinación (4 dds). Similares resultados han sido reportados por diversos autores en maíz y otros cultivos. Martínez (1999), comparó en condiciones de laboratorio el efecto del potencial del agua en la germinación de semillas de maíz de los cultivares Cargill 717 y Cargill 633, y encontró una inhibición total de la germinación en ambos cultivares bajo un potencial osmótico de -1.5 MPa, y una disminución en germinación por debajo de 
$50 \%$ en los dos cultivares bajo un potencial osmótico de -0.1 MPa. Además, el potencial de -1.5 MPa inhibió el crecimiento del coleóptilo en ambos cultivares. Méndez et al. (2002b), trabajaron con el efecto de soluciones osmóticas de manitol en la germinación de los cultivares de maíz Cargill 633, Himeca 2003 y Pioneer 3031, y apreciaron que en general, reducciones en los potenciales osmóticos causaron una disminución de la germinación, excepto a -3 MPa, donde el porcentaje de germinación fue superior al del control. La germinación fue más deprimida a -1.2 MPa, con reducciones de $95.84 \% ; 88.29 \%$ y 89.78\% para Cargill 633, Himeca 2003 y Pioneer 3031, respectivamente. Esta disminución del porcentaje de germinación con disminuciones del potencial osmótico del suelo, se debe al hecho de que existen tres etapas principales de la germinación: 1) activación; 2) digestión y translocación y 3) desarrollo de las plántulas, en la fase 1 de activación el volumen de la semilla se incrementa y esta caracterizada por la imbibición con cuatro subfases: 1) absorción de agua; 2) aumento de la respiración; 3) producción de enzimas y 4) elongación celular y emergencia de la radícula. En la fase de imbibición el incremento de la humedad es de 5-10\% en la semilla seca hasta 50\% después de la imbibición final y las semillas deben tener $40-60 \%$ de contenido de humedad para que ocurra la germinación, pero la absorción de agua es un balance del potencial osmótico en el suelo y el potencial osmótico en la semilla y las semillas deben tener un suministro uniforme de humedad. En el suelo, el potencial osmótico de la solución depende de la presencia de sales, con exceso de sales en el suelo se adquiere una fuerte presión negativa y el agua no entrará a la semilla, esta situación puede inhibir profundamente la germinación y llega a ser aún más problemática cuando el suministro de agua es bajo, porque el potencial osmótico del suelo llega a ser mayor. El estrés hídrico puede reducir el porcentaje de germinación, en algunos casos, la insuficiencia de agua inhibe la germinación de semillas de especies como lechuga, betarraga y apio, y la teoría es que estas semillas contienen inhibidores que necesitan ser lavados. Una menor germinación bajo condiciones de estrés hídrico puede ser atribuida al efecto de que las semillas aparentemente desarrollan una "latencia" osmóticamente forzada bajo condiciones de estrés hídrico, la cual puede ser una estrategia adaptativa de las semillas para prevenir la germinación bajo ambiente estresantes asegurando así el establecimiento apropiado de las plántulas (Singh et al., 1996; Prado et al., 2000).

En el presente trabajo los potenciales hídricos se ubicaron por encima del mínimo requerido para la germinación de las semillas de maíz, lo cual se desprende de la investigación efectuada por Hunter y Erickson (1952), al evaluar usando cinco tipos texturales de suelo los potenciales hídricos mínimos para la germinación de las semillas de maíz, soya, arroz y remolacha azucarera, y encontrarlos fijados en $-12.5 ;-6.6 ;-7.9$ y -3.5 atm, respectivamente, señalando que las diferencias observadas entre especies vegetales suelen atribuirse a que las fuerzas osmóticas internas para la imbibición del agua son distintas; esto ha sido confirmado por Briede y McKell (1992). Dado que en el presente trabajo los potenciales hídricos superaron el mínimo requerido para la germinación de las semillas de maíz, es de presumir que este factor no fue una limitante importante en este proceso, lo cual parece corroborarse en el hecho de que la interacción potencial osmótico $\mathrm{x}$ cultivar coincide en mostrar que únicamente los porcentajes de germinación disminuyeron en ambos cultivares respecto al control en el potencial osmótico de -1.2 MPa.

Diferencias en la germinación entre cultivares de maíz por efecto de estrés hídrico han sido 
reportadas. Zeid y El-Semary (2001) indicaron que la germinación de las semillas de Giza 2 se redujo $60 \%$ en el tratamiento a $-0.6 \mathrm{MPa}$ a $50 \%$ a $-0.8 \mathrm{MPa}$, mientras la reducción en la germinación del genotipo susceptible a la sequía SCH 155 comenzó a -0.2 MPa (80\%) y disminuyó progresivamente y registró $0 \%$ a 0.8 MPa. Méndez et al., (2002a), trabajaron con soluciones de glucosa y su efecto en tres cultivares de maíz (Cargill 633, Himeca 2003 y Pioneer 3031), y observaron que el porcentaje de germinación se incrementó $2.05 \%$ y $3.76 \%$ en Cargill 633 e Himeca 2003, respectivamente, en el potencial de - $0.3 \mathrm{Mpa}$, pero en Pioneer 3031 no se vio afectado, las mayores reducciones en la germinación ocurrieron a -1.2 Mpa con $88.52 \%$; $94.41 \%$ y $87.50 \%$ para Cargill 633, Himeca 2003 y Pioneer 3031, respectivamente.

El tamaño de la semilla no afectó al proceso de germinación en las condiciones de estrés hídrico creadas en este trabajo. Resultados similares fueron indicados por Mian y Nafziger (1992) quienes reportaron que el tamaño de la semilla no tuvo efecto sobre el porcentaje de germinación en trigo (Triticum aestivum). Aunque no se presentó influencia del tamaño de la semilla en la germinación por efecto del potencial osmótico, otros investigadores trabajando con potenciales osmóticos menores a los empleados en esta investigación señalan ventajas de las semillas de menor tamaño respecto a las mayores en tal condición. Muchena y Grogan (1977), quienes estudiaron el efecto del tamaño de la semilla del cultivar White Cloud (cotufero), y las líneas R181 y Ay499 sobre la germinación del maíz bajo condiciones simuladas de estrés de agua usando distintas concentraciones de manitol $(0,-10,-14$ y $-18 \mathrm{~atm})$, encontraron que la germinación de la semillas pequeñas y medianas de White Cloud fue significativamente más alta que la de las semillas grandes a -14 atm $(90.9 \%$ y $85.2 \%$ para la semilla pequeña y mediana, respectivamente, y $67.4 \%$, para la grande) y 18 atm $(43.6 \%$ y $34.5 \%$ semilla pequeña y mediana, respectivamente, y $19.8 \%$ la grande), pero no a 0 y -10 atm $(99.4 \%, 100 \%$ y $100 \%$ para los tamaños pequeño, mediano y grande, respectivamente). Esto sugiere, de acuerdo con los autores, que la ventaja de las semillas pequeñas, en términos de germinación bajo condiciones de estrés de agua, se incrementa cuando el potencial del agua del medio es artificialmente disminuido. Aun cuando no hubo diferencias significativas entre el porcentaje de germinación de la fracción de semillas pequeñas y la fracción de semillas grandes de White Cloud en todos los niveles de concentración de manitol, la fracción de semillas medianas fue casi siempre intermedia en porcentaje de germinación entre la fracción pequeña y la fracción grande $(98.4 \%, 93.5 \%$ y $97.8 \%$, para los tamaños pequeño, mediano y grande, respectivamente, a $-10 \mathrm{~atm} ; 90.9 \%$, $85.2 \%$ y $67.4 \%$, a -14 atm, y $43.6 \%, 34.5 \%$ y $19.8 \%$, a -18 atm). Hubo diferencias significativas entre las tres facciones de semilla de R181 a -10 atm, pero no a mayores niveles de concentración de manitol. En Ay499, los resultados fueron menos consistentes; a -18 atm solamente germinó la fracción de semillas pequeñas. Muchena y Grogan (1977), señalan que las diferencias en la germinación entre diferentes tamaños de semillas dentro de los cultivares de maíz por efecto de condiciones simuladas de estrés de agua usando distintas soluciones osmóticas, pueden ser atribuidas exclusivamente a factores no genéticos, si se asume que no existen diferencias genéticas entre las fracciones de semillas dentro de un tipo de maíz. Las diferencias en peso dentro de un tipo resultan de los factores ambientales y el efecto de la posición en la mazorca. En consecuencia, el tamaño de la semilla ejerce influencia en el porcentaje de germinación de las semillas de maíz bajo condiciones simuladas de estrés de agua. 


\section{CONCLUSIONES}

- Las tensiones de humedad logradas a través de las frecuencias de riego permitirían seleccionar cultivares tolerantes a esta condición de estrés en el proceso de germinación con una tensión de la humedad del agua en el suelo de -1,2 MPa, presentando una mayor tolerancia el cultivar Pioneer 3031 respecto a Himeca 95.

- El tamaño de la semilla no influenció el proceso de germinación en ninguna de las condiciones de estrés en estudio.

\section{BIBLIOGRAFÍA}

Ávila, M. ; De Lucca e Braccini, A. y Scapim, C. 2007. Teste de comprimento de plântulas sob estresse hídrico na avaliação do potencial fisiológico das sementes de milho. Revista Brasileira de Sementes 29 (2):117-124

Azcon-Bieto, J. y Talon, M. 1993. Fisiología y Bioquímica Vegetal. Interamericana McGraw-Hill, Madrid, p537-539

Briede, J. y Mckell, C. 1992. Germination of seven perennial arid land species, subjected to soil moisture stress. Journal of Arid Environments 23:263-270

Cornaglia, P.; Schrauf, G.; Nardi, M. y Deregibus, V. 2005. Emergence of Dallisgrass as affected by soil water availability. Rangeland Ecology Management 58:35-40

Edmeades, G.; Bolaños, J. y Lafitte, H. 1992. Progress in breeding for drought tolerance in maize. En: Wilkinson, D. (Ed). Proceedings of 47th Annual Corn and Sorghum International Research Conference, Chicago, Asta p93-111

Ellis, R. y Roberts, E. 1980. Towards a national basis for testing seed quality. En: Hebblethwaite, P. (Ed). Seed Production, Butterworths, Londres, p605-635
Gazanchian, A.; Khosh Kholgh Sima, N.; Malboobi, M. y Majidi Heravan, E. 2006. Relationships between emergence and soil water content for perennial cool-season grasses native to Iran. Crop Science 46:544-553

Hartmann, H.; Kester, D. y Davies, T. 1993. Plant Propagation Principles and Practices. Prentice-Hall, New Delhi, 647 pp.

Hunter, J. y Erickson, A. 1952. Relation of seed germination to soil moisture tension. Agronomy Journal 44:107-109

Khan, M. y Ungar, I. 1984. The Effect of salinity and temperature on the germination of polymorphic seeds and growth of Atriplex triangularis Willd. American Journal of Botany 71(4):481-489

Martínez A. 1999. Efecto de la temperatura y del contenido de agua del suelo en la germinación y crecimiento inicial en dos cultivares de maíz (Zea mays L.) con diferentes contenidos de humedad inicial en las semillas. Tesis M.Sc., Universidad de Oriente, Monagas

Méndez, J.; Ibarra, P. y Merazo P. 2002a. Germinación de semillas y desarrollo de plántulas de tres híbridos de maíz 
(Zea mays L.) bajo soluciones osmóticas III. Glucosa. VI Festival del Maíz y VI Jornada Científica Nacional del Maíz, Maracay. http:// www.ceniap.gov.ve/pbd/Congresos/ jornada s \% $20 \mathrm{de} \% 20 \mathrm{~m}$ a i z / $6 \% 20 j o r n a d a s / c a r t e l e s / t e c n o s e m i l l a /$ jmendezglucosa.htm. [Accedido: 04/ 12/2007]

Méndez, J.; Ibarra, P. y Merazo P.. 2002b. Germinación de semillas y desarrollo de plántulas de tres híbridos de maíz (Zea mays L.) bajo soluciones osmóticas IV. Manitol. VI Festival del Maíz y VI Jornada Científica Nacional del Maíz, Maracay. http:// www.ceniap.gov.ve/pbd/Congresos/ jorna da s \% $20 \mathrm{de} \% 20 \mathrm{ma} \mathrm{iz/}$ 6\%20jornadas/carteles/tecnosemilla/ jmendezmanitol.htm. [Accedido: 04/ 12/2007]

Mian, M. y Nafziger, E. 1992. Seed size effect on emergence, head number and grain yield of winter wheat. Journal of Production Agricultural 5:265-268

Muchena, S. y Grogan, C. 1977. Effects of seed size on germination of corn (Zea mays) under simulated water stress conditions. Canadian Journal of Plant Sciences 57:921-923
Musgrove, M.; Priestley, D. y Leopold, A. 1980. Methanol stress as a test of seed vigor. Crop Science 20(5):626-630

Prado, F.; Boero, C.; Gallardo, M. y Gónzalez, J. 2000. Effect of $\mathrm{NaCl}$ on germination, growth and soluble sugar content in Chenopodium quinoa wild seeds. Botanical Bulletin of Academia Sinica $41: 27-34$

Richard, S. 1967. Water release as soil property relating to the use of water by plants. Transaction of the ASAE. $45^{\text {th }}$ Annual Meating Riverside, California, 15pp.

Singh, P.; Bhaglal, P. y Bhullar, S. 1996. Differential levels of wheat germ agglutinin (WGA) in germinating embryos of different wheat cultivars in response to osmotic stress. Plant Physiology Biochemistry 34:547-552

Turk, M.; Rahman, A.; Tawaha, M. y Lee. K. 2004. Seed germination and seedling growth of three lentil cultivars under moisture stress. Asian Journal of Plant Sciences 3(3):394-397

Zeid, I. y El-Semary, N. 2001. Response of two differentially drought tolerant varieties maize to drought stress. Pakistan Journal of Biological Sciences (7):779784 